\title{
The expression and meaning of CD68, CD163, CD57, and IgG4 in granulomatous lobular mastitis
}

\author{
Cheng Kong', Chaojie Zhang ${ }^{2}$, Yaqin $\mathrm{Wu}^{2}$, Zheng Zeng ${ }^{2}$, Hong Yu ${ }^{3}$, Jie Zeng ${ }^{2}$, Shanshan Lei ${ }^{2}, \mathrm{Jie}^{\mathrm{He}^{2}}$, \\ Peizhi Fan ${ }^{2}$ \\ ${ }^{1}$ Department of General Surgery, Hunan Provincial People's Hospital Xingsha Branch (People's Hospital of Changsha County), Changsha 410100, \\ China; ${ }^{2}$ Department of Breast and Thyroid Surgery, Hunan Provincial People's Hospital/The First Affiliated Hospital of Hunan Normal University, \\ Changsha 410005, China; ${ }^{3}$ Department of Pathology, The Third People's Hospital of Shenzhen, Shenzhen 518116, China \\ Contributions: (I) Conception and design: C Kong; (II) Administrative support: C Zhang; (III) Provision of study materials or patients: J Zeng, J He, \\ S Lei; (IV) Collection and assembly of data: Z Zeng, C Kong, H Yu; (V) Data analysis and interpretation: C Kong, P Fan, Y Wu; (VI) Manuscript \\ writing: All authors; (VII) Final approval of manuscript: All authors. \\ Correspondence to: Chaojie Zhang; Yaqin Wu. Department of Breast and Thyroid Surgery, Hunan Provincial Peoples Hospital/The First Affiliated \\ Hospital of Hunan Normal University, Changsha 410005, China. Email: zhangchaojie@hunnu.edu.cn; wuyaqin1996@163.com.
}

Background: The exact etiology and pathogenesis of granulomatous lobular mastitis (GLM) are yet to be illuminated. This study aimed to investigate CD68, CD163-positive M2 macrophages, CD57-positive natural killer (NK) cells, and IgG4 in GLM lesion tissue to explore their correlation with the occurrence and clinical features of GLM.

Methods: Surgical pathologic specimens of GLM were collected from patients admitted to Hunan Provincial People's Hospital between October, 2014 and October 2015. Based on the postoperative pathological diagnosis, the tissues were divided into 3 groups: the experimental group (GLM, n=36), control group 1 (plasma cell mastitis, PCM, n=17), and control group 2 (breast cystic hyperplasia, $\mathrm{n}=10$ ). Immunohistochemical staining was carried out using Elivision super testing to detect CD68, CD163, CD57, and IgG4 expression in the pathological tissue samples. The relationship between clinical parameters, including age, reproductive condition, nipple retraction, and tumor size, and the expressions of CD68, CD163, CD57, and IgG4 was analyzed.

Results: There was no obvious difference in the levels of CD68, CD163, and CD57 expression between the GLM group and the PCM group, although both groups had higher expression levels of expression than the breast cystic hyperplasia group $(\mathrm{P}<0.05)$. In the GLM group, the expression level of CD57 at 2 weeks3 months was significantly higher than at $\leq 2$ weeks $(\mathrm{P}<0.05)$. The expression level of CD57 in PCM patients $>2$ years after lactation was significantly higher than in patients $\leq 2$ years after lactation $(\mathrm{P}<0.05)$. The level of IgG4 expression in GLM patients with nipple retraction was significantly higher than in those without nipple retraction $(\mathrm{P}<0.05)$.

Conclusions: Inflammatory cells are closely linked to the occurrence of GLM and PCM. In our study, both the GLM and PCM groups had low expression of IgG4, but the expression level of IgG4 in GLM patients with inverted nipples was significantly higher than that in patients without inverted nipples. This suggests that there may be two different clinical subtypes of GLM. Furthermore, our research also found that NK cells can provide a basis for GLM clinical staging.

Keywords: Granulomatous lobular mastitis (GLM); plasma cell mastitis (PCM); immunohistochemical; M2 macrophages; natural killer cell (NK cells); IgG4-positive plasmocyte

Submitted Feb 13, 2020. Accepted for publication Jun 04, 2020.

doi: 10.21037/gs-20-419

View this article at: http://dx.doi.org/10.21037/gs-20-419 


\section{Introduction}

Granulomatous lobular mastitis (GLM), which takes its name from tumor samples of idiopathic granulomatous mastitis, is a chronic inflammatory condition that affects the breast. GLM often occurs on one side of the breast, although lesions sometimes present in both sides, mostly in the areola area and the upper outer quadrant. In some patients, multiple quadrants or even the whole breast may be affected. GLM mainly manifests as a single hard mass in the breast, and is usually accompanied by no pain or slight pain. The boundary of GLM is not clear and the surface is not smooth. The mass may adhere to the skin or surrounding tissue. The skin surrounding the affected area can appear red and swollen. GLM can be accompanied by ipsilateral axillary lymph node enlargement and does not have any obvious systemic symptoms.

The etiology and pathogenesis of GLM are not fully understood. The following factors have been identified in many studies as contributing to the disease: corynebacterium infection (1); long-term oral psychoactive drugs and short-acting contraceptives (2); hyperprolactinemia (3); trauma (4); milk-induced hypersensitivity, or pregnancy or milk siltation $(5,6)$; and autoimmune correlation (7). GLM is difficult to diagnose, and with a $50 \%$ recurrence rate, effective treatment also poses a challenge (8). Histopathological examination is the golden criteria of diagnosis. Histologically, GLM is characterized by lobular granulomas composed of macrophages, neutrophils, lymphocytes, monocytes, and non-cheese-like necrotic lesions. Microscopically, granulomas are characterized by large numbers of Langhan's giant cells, neutrophils polymorphs, and epithelioid histocytes. In addition, microabscesses, necrosis, sinus tracts, and duct ectasia can also be found (9). At present, no standard guidelines exist to guide the treatment of GLM.

At present, GLM is widely seen as an autoimmune disease, and experiments related to the abnormal immune inflammation index have confirmed that the degree of immune disorder differs according to age, family status, and breast disease-related inflammatory agglomeration area (10). However, its association with M2 macrophages, natural killer (NK) cells, or IgG4-positive plasmocytes has not been ascertained.

Clinically, PCM and GLM are difficult to differentiate, and their diagnosis and treatment are also complicated. The diagnosis of both depends on histopathological examination. As a common benign disease of the breast, breast cystic hyperplasia has a completely different pathogenesis from inflammatory diseases. Therefore, patients with PCM and breast cystic hyperplasia were selected as the control group to help distinguish GLM, PCM, and common benign diseases.

There are two types of macrophages. The second type, activated macrophages, also known as M2-type macrophages, are predominantly characterized as CD68+ (pan macrophages marker) and CD163+ (M2 specific marker). M2-type macrophages can dampen the immune response and limit inflammation (11). To explore the significance of CD68+, CD163-positive M2 macrophages, CD57-positive NK cells, IgG4-positive plasmocytes in relation to lactation mastitis morbidity, this study used immunohistochemical to test the expression of CD68, CD163, CD57, and IgG4 in GLM, PCM, breast cystic hyperplasia patients. In this pilot study, we examined changes in inflammatory cells and cytokines to further explore the pathogenesis of GLM, with the ultimate aim of reducing the damage caused by breast surgery and providing a theoretical basis for future treatment of the disease.

\section{Methods}

The trial was conducted in accordance with the Declaration of Helsinki (as revised in 2013) and the Harmonized Tripartite Guideline for Good Clinical Practice from the International Conference on Harmonization. This study was reviewed and approved by the Hamilton Integrated Research Ethics Board (approval \#2017SK2142) and the Hoffmann-La Roche global review committee. All patients enrolled completed the informed consent form.

\section{Clinical materials}

\section{Experimental group}

The study enrolled 36GLM patients who underwent mammary and thyroid surgery between October, 2014 and October, 2015 at Hunan Province People's Hospital (the First Affiliated Hospital of Hunan Normal University). The diagnoses were confirmed by postoperative pathological histology. All of the patients were female, married, and aged between 25 and 52 (mean, 33.25 \pm 6.5 ) years. None of the patients were pregnant or lactating. The average duration of the condition was 2.4 months (range, 1 day to 1 year).

\section{Control group 1}

Control group 1 comprised 17 cases of plasma cell mastitis 
(PCM) patients who underwent mammary gland thyroid surgery at our hospital during the same period. All of the diagnoses were confirmed by postoperative pathology. The patients were aged between 22 and 39 (mean, 29.18 5.71) years old.

\section{Control group 2}

Control group 2 comprised 10 cases of breast cystic hyperplasia, as confirmed by postoperative pathological histology. The patients were aged between 24 and 43 (mean, $33.5 \pm 7.95$ ) years old.

There was no significant difference in age between the experimental group and the 2 control groups $(\mathrm{P}>0.05)$.

\section{Inclusion and exclusion criteria}

All specimens were confirmed after reading by 2 or more experienced clinical pathology doctors. The clinical and pathological data of the specimens had to be intact, and the patients should have been followed-up on a regular basis. Cases with malignant breast tumors were excluded from the study, as were those with diabetes, rheumatism, and autoimmune diseases, such as systemic lupus erythematosus (SLE). Patients with liver or kidney disease, tuberculosis, and other medical problems were excluded. Finally, participants who had a history of pregnancy or lactation in the 6 months before surgery were excluded.

\section{Research methods}

Paraffin-embedded specimens were collected from October 2014 to October 2015. The EliVision super method of immunohistochemical staining was used. A negative control was used throughout the experiment. PBS was used instead of a negative resistance. The expression levels of CD68, CD163、CD57, and IgG4 were observed semi-quantitatively (12-14). Comparisons were made based on: (I) age (10) : baby boomers (aged 25 to 34) and non-baby boomers: (aged 21 to 24 and 35 to 55); (II) fertility condition (10): around lactation: paroxysm $<2$ years after childbirth, and after lactation: paroxysm $\geq 2$ years after childbirth; and (III) nipple depression (15): moderate (the nipple retraction technique can be used to pull reset, after the release of the nipple is retracted), severe (nipple retraction technique cannot be used to pull reset, and no nipple invagination), normal nipple or mild invagination (nipple can use the technique to pull reset, loosen the nipple no retraction, but after the compression of the nipple still retract). Based on the common clinical inflammatory disease progression installment method, the following phases were considered: the acute phase ( 2 weeks or less), the subacute phase ( 2 weeks to 3 months), and the chronic phase (more than 3 months). Sectionalization was done by lesion size: $\leq 3,3-6$, and $\geq 6 \mathrm{~cm}$.

\section{Statistical analysis}

Statistical analyses were carried out by SPSS 19.0 (IBM Corporation, Armonk, NY, USA) statistical package. Score data used $\bar{x} \pm \mathrm{S}$. If the variance was neat, Fisher's LSD test for pairwise comparison of one-way anova was adopted. If the variance was not homogeneous, the nonparametric multi-group Kruskal-Wallis $\mathrm{H}$ test was adopted. Inspection level was for $\alpha=0.05$ (bilateral) or $\alpha^{\prime}=0.0083$ (one-sided).

\section{Results}

The expression of CD68 and CD163-positive M2 macrophages, CD57-positive NK cells and IgG4-positive plasma cells in the experimental group and control groups

(I) CD68 and CD163-positive M2 macrophages and IgG4-positive plasma cells were detected in the cytoplasm, CD57-positive NK cells were detected in the cell membranes. M2 macrophages, NK cells and IgG4-positive plasma cells were detected in the GLM and PCM groups, and there was no obvious difference between the two groups (Figures 1,2). There was no positive expression in the breast cystic hyperplasia group, and the difference was statistically significant compared with GLM and PCM (Table 1).

(II) There were significant expressions of inflammatory cells (CD68 and CD163-positive M2 macrophages, CD57-positive NK cells and IgG4-positive plasma cells) detected in the experimental group and control groups $(\mathrm{P}<0.05)$. There was no obvious difference between expression in the GLM and PCM groups, and both had higher expression than in the breast cystic hyperplasia group $(\mathrm{P}<0.05)$ (Table 2).

\section{The relationship between expression of inflammatory cells and clinical parameters in the experimental group and control group 1}

\section{Test index comparison of two different courses}

(I) Non-parametric Kruskal-Wallis $\mathrm{H}$ test was used to compare CD68, CD163, and IgG4 expression in the 

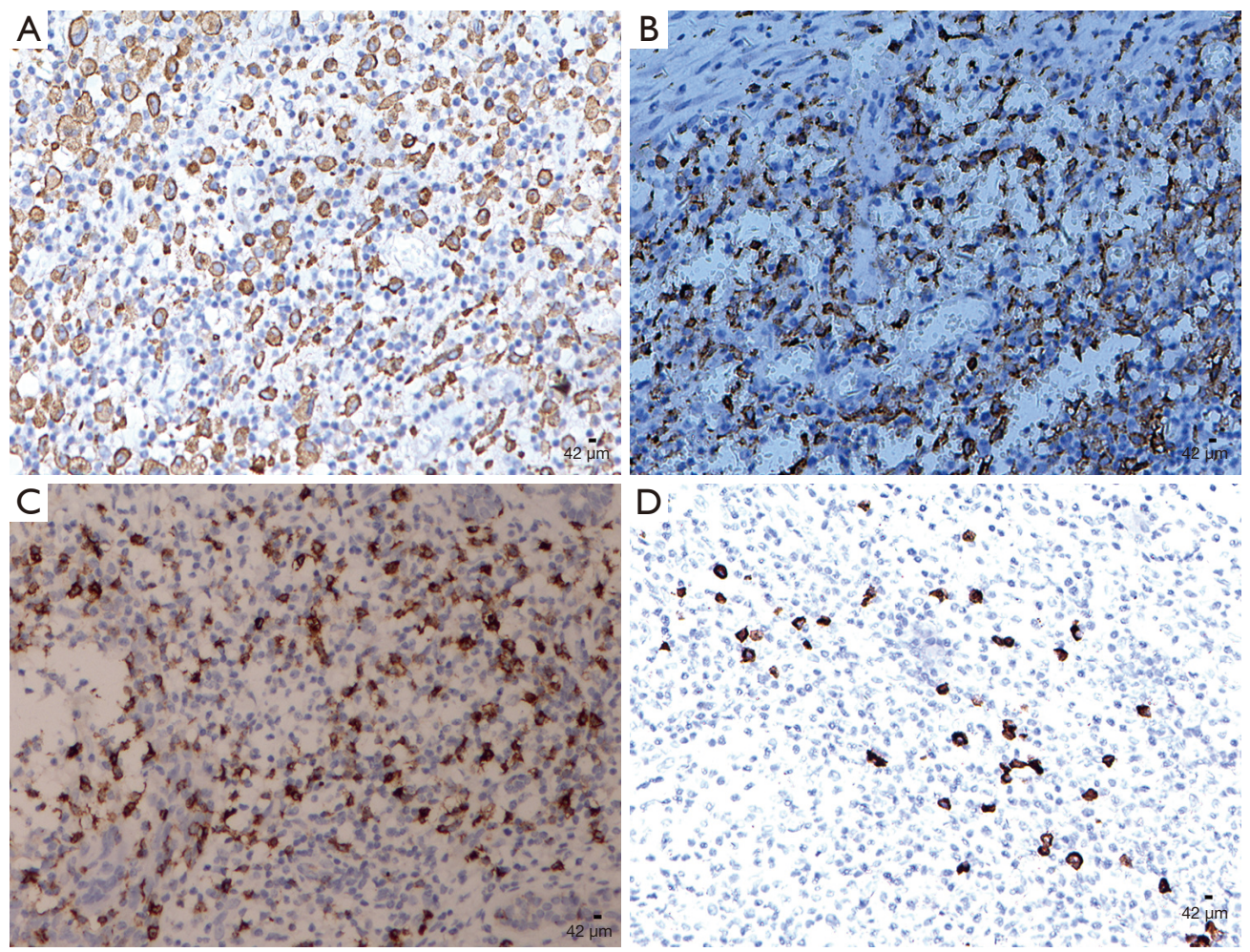

Figure 1 Immunohistochemistry staining from the GLM group: (A) CD68 200×, (B) CD163 200×, (C) CD57 200×, and (D) IgG4 staining 200x. GLM, granulomatous lobular mastitis.

GLM group at each stage of the disease course, and no significant statistical difference was found $(\mathrm{P}>0.05)$. The expression level of CD57 in the GLM group at 2 weeks-3 months was significantly higher than that at $\leq 2$ weeks, with significant statistical difference $(\mathrm{P}<0.05)$. No significant change was observed at $\geq 3$ months $(\mathrm{P}>0.05)$ (Table 3).

(II) At various stages of the disease courser showed no significant statistical difference between the four indicators of $\mathrm{PCM}(\mathrm{P}>0.05$, Table 4).

(III) NK cells in the GLM group at the acute stage ( $\leq 2$ weeks), and chronic phase ( $\geq 3$ months) was lower than that in the PCM group, and revealed a widening gap between the two groups (Figure 3). IgG4 expression in the PCM group gradually increased at chronic phase ( $\geq 3$ months), and the gap started to widen (Figure 4). There was no obvious difference between macrophages in the GLM and PCM groups at the different stages of the disease course (Figure 5).

\section{Layered detection index comparison of different ages} The expressions of CD68 and CD163-positive M2 macrophages, CD57-positive NK cells and IgG4-positive plasma cells were compared between baby boomers and non-baby boomers both in the GLM and PCM groups by nonparametric Kruskal-Wallis $\mathrm{H}$ test (Table 5) and analysis of variance (Table 6). There was no significant statistical difference [MOU1] in expression between baby boomers and non-baby boomers $(\mathrm{P}>0.05)$.

\section{Holding detection indicators in different fertility status to compare of the disease}

The expression of CD68, CD163 and IgG4 between paroxysm $<2$ years after childbirth and paroxysm $\geq 2$ years after childbirth both in GLM and PCM groups was compared using the nonparametric Kruskal-Wallis $\mathrm{H}$ test and analysis of variance, There was no significant statistical difference in expressions between the two groups $(\mathrm{P}>0.05$, Tables 7,8). 

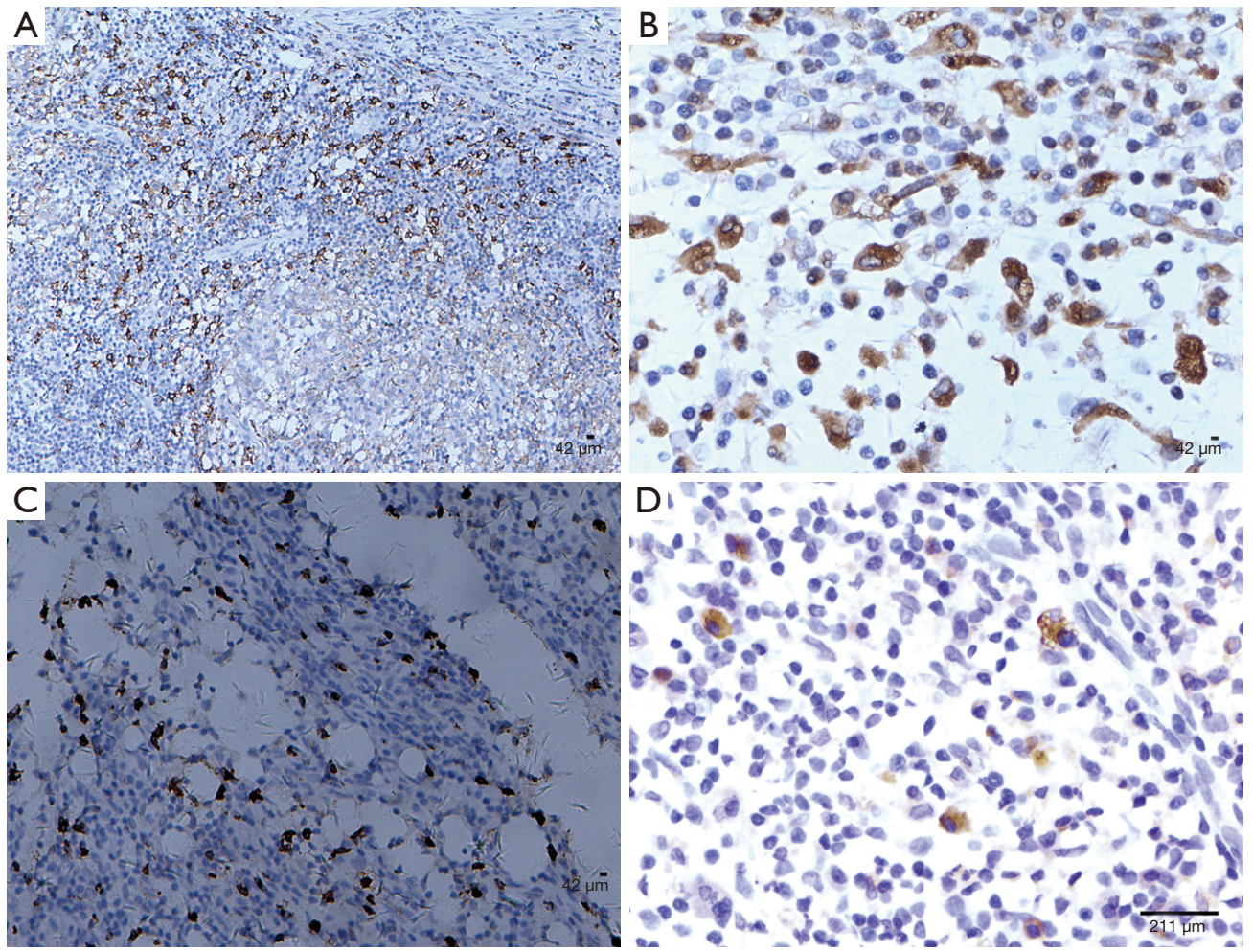

Figure 2 Immunohistochemistry staining from the PCM group: (A) CD68 200×, (B) CD163 200×, (C) CD57 200×, and (D) IgG4 staining 400×. PCM, plasma cell mastitis.

Table 1 Comparison between the expression rates of CD68, CD163, CD57, and IgG4 in the experimental and control groups (\%)

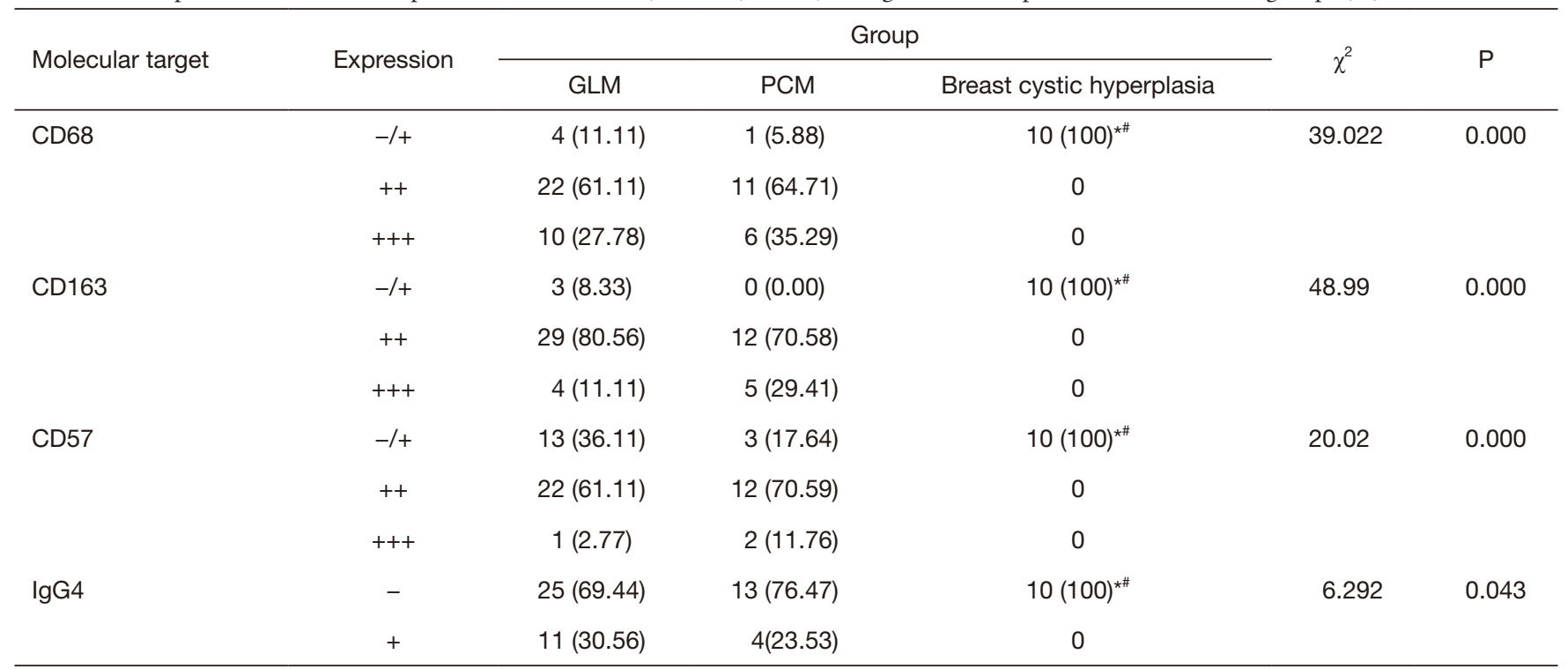

With granulomatous lobular mastitis * is $\mathrm{P}<0.05$, and plasma cell mastitis ${ }^{\#}$ is $\mathrm{P}<0.05$. GLM, granulomatous lobular mastitis; PCM, plasma cell mastitis. 
Table 2 Comparison between the quantities of CD68, CD163, CD57, and IgG4 in the experimental and control groups

\begin{tabular}{|c|c|c|c|c|c|c|c|c|}
\hline Items & \multicolumn{2}{|l|}{ GLM } & \multicolumn{2}{|l|}{ PCM } & \multicolumn{2}{|c|}{ Breast cystic hyperplasia } & Z & $\mathrm{P}$ \\
\hline Age & $33.25 \pm 6.50$ & 32.0 & $29.18 \pm 5.71$ & 27.0 & $33.50 \pm 7.95$ & 32.00 & 4.354 & 0.226 \\
\hline CD68 & $4.31 \pm 1.82$ & 5.00 & 4. $82 \pm 1.47$ & 5.0 & $1.90 \pm 1.10^{\star \#}$ & 2.00 & 22.504 & $<0.001$ \\
\hline CD163 & $4.25 \pm 1.30$ & 4.00 & $4.71 \pm 1.36$ & 5.0 & $0.60 \pm 0.97^{\star \#}$ & 0.00 & 25.993 & $<0.001$ \\
\hline $\operatorname{lgG} 4$ & $6.80 \pm 6.51$ & 5.88 & $8.12 \pm 8.05$ & 6.80 & $3.60 \pm 2.07$ & 4.00 & 22.85 & $<0.001$ \\
\hline
\end{tabular}

With granulomatous lobular mastitis * is $\mathrm{P}<0.05$, and plasma cell mastitis ${ }^{\#}$ is $\mathrm{P}<0.05$. GLM, granulomatous lobular mastitis; PCM, plasma cell mastitis.

Table 3 Comparison between the expressions of CD68, CD163, CD57, and IgG4 at three different stages of GLM

\begin{tabular}{|c|c|c|c|c|c|c|c|c|}
\hline Items & \multicolumn{2}{|c|}{2 weeks or less } & \multicolumn{2}{|c|}{2 weeks -3 months } & \multicolumn{2}{|c|}{ More than 3 months } & Z & $\mathrm{P}$ \\
\hline CD68 & $4.07 \pm 2.056$ & 5.00 & $4.73 \pm 1.44$ & 4.00 & $3.86 \pm 2.12$ & 5.0 & 0.465 & 0.792 \\
\hline CD163 & $4.29 \pm 1.49$ & 4.00 & $4.33 \pm 1.23$ & 4.00 & $4.00 \pm 1.156$ & 4.0 & 0.934 & 0.627 \\
\hline CD57 & $17.51 \pm 14.73$ & 19.6 & $40.00 \pm 21.8^{\star}$ & 36.2 & $24.91 \pm 13.35$ & 17.0 & 7.122 & 0.028 \\
\hline
\end{tabular}

Compared with 2 weeks or less * $\mathrm{P}<0.05$, compared with 2-3 months ${ }^{\#} \mathrm{P}<0.05$. GLM, granulomatous lobular mastitis; PCM, plasma cell mastitis.

Table 4 Comparison between the expressions of CD68, CD163, CD57, and IgG4 at three different stages of PCM

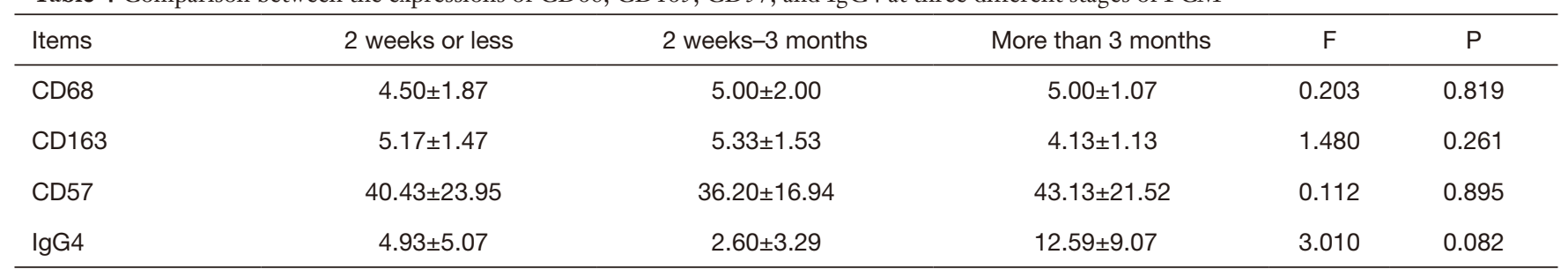

PCM, plasma cell mastitis.

\section{PCM patient childbirth}

The level of CD57 expression was significantly higher in the group who experienced GLM onset $>2$ years after childbirth than in the group with onset $\leq 2$ years after childbirth $(\mathrm{P}<0.05$, Table 8$)$.

\section{Comparison between patients with or without nipple retraction}

The expressions of CD68, CD163, and CD57 were compared between patients with and patients without nipple retraction in the GLM and PCM groups using nonparametric Kruskal-Wallis $\mathrm{H}$ rank and inspection and analysis of variance. No significant statistical difference was found in CD68, CD163, and CD57 expression between patients with and without nipple retraction $(\mathrm{P}>0.05$, Tables 9,10).

The expression level of IgG4 in the GLM group was significantly higher in patients without nipple retraction, and there were statistically significant differences $(\mathrm{P}<0.05$, Table 9). 


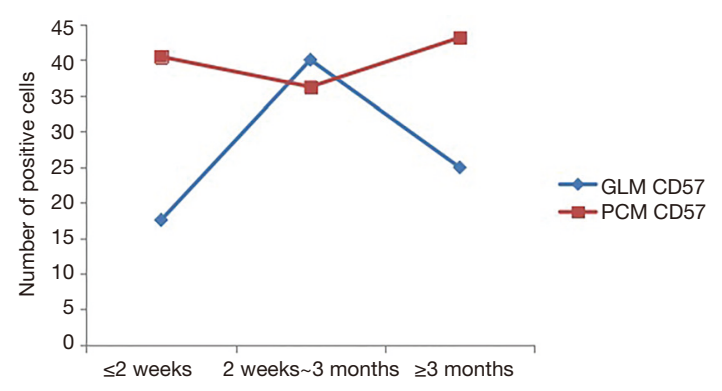

Figure 3 Comparison of the expression levels of CD57 in the GLM and PCM groups. GLM, granulomatous lobular mastitis; PCM, plasma cell mastitis.

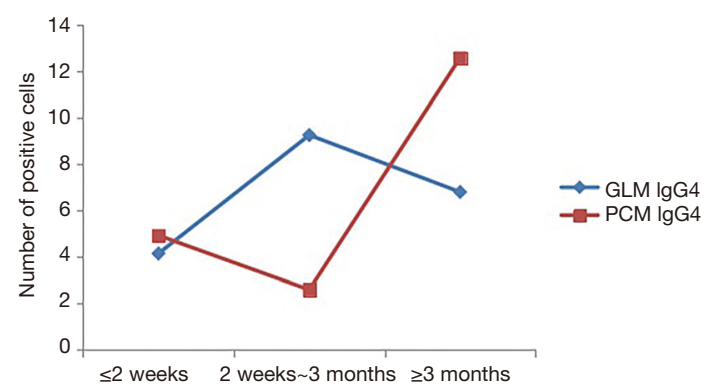

Figure 4 Comparison of the expression levels of IgG4 in the GLM and PCM groups. GLM, granulomatous lobular mastitis; PCM, plasma cell mastitis.

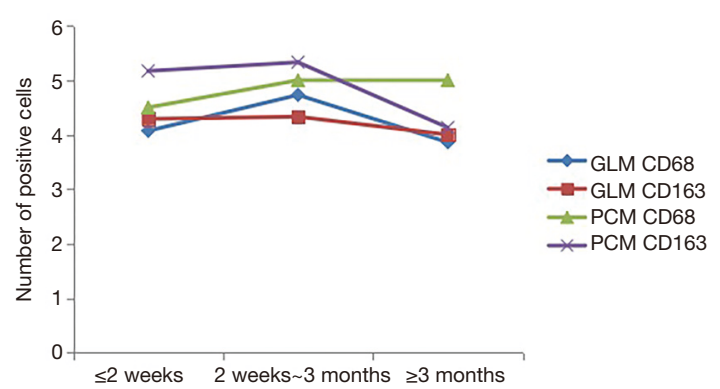

Figure 5 Comparison of the expression levels of CD68 and CD163 in the GLM and PCM groups. GLM, granulomatous lobular mastitis; PCM, plasma cell mastitis.

\section{Comparison of different lesion ranges}

The expression of CD68, CD163, CD57, and IgG4 both in GLM group between different lesion size $(\leq 3,3-6$, and $\geq 6 \mathrm{~cm}$ ) was consistent. No significant statistical difference was observed $(\mathrm{P}>0.05$ Tables 11,12).

\section{Discussion}

GLM, PCM, subareolar abscess (Zuska disease), and lymphocytic sclerosing lobular mastitis, are collectively called non-lactational mastitis. GLM was previously considered to be a rare benign inflammatory disease of the breast, which occurs in the non-lactational period, and its incidence has increased considerably in recent years. European and American countries have reported that non-lactational mastitis accounts for $3 \%$ of benign breast disease (15). Our team found that non-lactational mastitis accounted for about $10.5 \%$ of the total surgeries for benign breast diseases at the hospital during the same period (16), among which procedures for GLM and PCM were the most common.

In recent years, GLM has been reported more frequently in the Asia-Pacific region, and there have also been higher rates of GLM recorded among Asian and Hispanic women (17). Therefore, greater attention should be given to the disease in these regions.

It has been reported (18) that the misdiagnosis rate with GLM is as high as $40 \%$, and the disease needs to be distinguished from inflammatory breast cancer, breast cyst and infection, Paget's disease, and lactational mastitis. GLM clinically manifests as aperiodic primary breast pain, nipple discharge, pull invagination, non-lactating breasts, centripetal developing scleroderma, abscess, skin redness on the side of the breast, swelling, axillary lymph node enlargement, and sometimes an "orange peel" appearance. However, GLM is clinically difficult to differentiate from inflammatory breast cancer and non-lactational specific breast inflammatory diseases such as breast tuberculosis. Its clinical stage can be divided into the mass, abscess, and ulceration stages, but there is no obvious boundary between the three. Clinical staging is of great importance to the surgery, affecting the scope of the surgery, surgical methods, postoperative plastic effect, and postoperative psychological recovery of patients. Some domestic scholars do not believe that the clinical stages are the same disease at different stages of development, but rather they believe that, similar to the different molecular classification in breast cancer, granulomatous mastitis has different types, based on the expression of inflammatory factors, and so treatment plans and prognosis are not consistent.

In 1972, when the disease was originally reported by Kesler, it was reported as being self-limiting (8), while 
Table 5 Comparison between the expressions of CD68 and CD163 (GLM group only) and IgG4 (GLM and PCM groups) in baby boomers and non-baby boomers

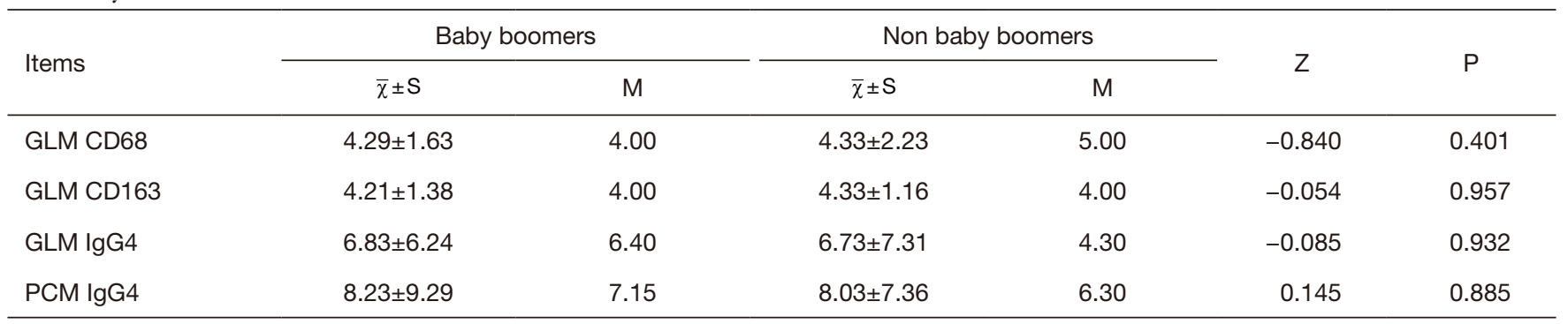

Statistically significant difference $(\mathrm{P}<0.05)$. GLM, granulomatous lobular mastitis; PCM, plasma cell mastitis.

Table 6 Comparison between the expressions of CD68 and CD163 (PCM group only) and CD57 (GLM and PCM groups) in baby boomers and non-baby boomers

\begin{tabular}{lcccc}
\hline Items & Baby boomers & Non-baby boomers & $F$ & P \\
\hline GLM CD57 & $30.59 \pm 21.30$ & $23.78 \pm 17.85$ & 0.951 & -1.205 \\
PCM CD68 & $4.38 \pm 1.3020$ & $5.22 \pm 1.56$ & 0.122 & 0.247 \\
PCM CD163 & $4.75 \pm 1.28$ & $4.67 \pm 1.50$ & -0.206 & 0.904 \\
PCM CD57 & $39.83 \pm 22.09$ & $41.96 \pm 20.47$ & 0.839 \\
\hline
\end{tabular}

Statistically significant difference $(\mathrm{P}<0.05)$. PCM, plasma cell mastitis; GLM, granulomatous lobular mastitis.

Table 7 Comparison of CD68, CD163, and IgG4 expressions between groups based on time of onset after childbirth in the GLM group

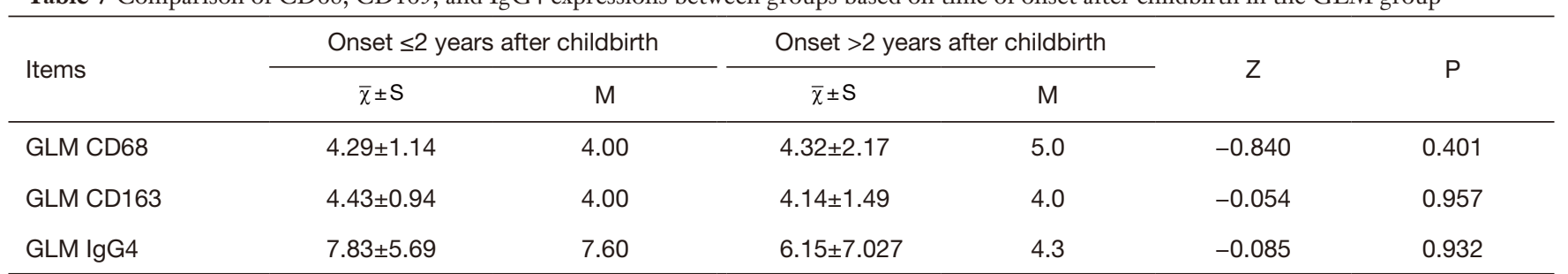

Statistically significant difference $(\mathrm{P}<0.05)$. GLM, granulomatous lobular mastitis.

Table 8 Comparison of the expressions of CD57 (GLM and PCM groups) and CD68, CD163, and IgG4 (PCM group only) between groups based on time of onset after childbirth in the GLM group

\begin{tabular}{|c|c|c|c|c|}
\hline Items & Onset $\leq 2$ years after childbirth & Onset $>2$ years after childbirth & $\mathrm{F}$ & $P$ \\
\hline PCM CD68 & $4.89 \pm 1.05$ & $4.75 \pm 1.91$ & 0.189 & 0.853 \\
\hline PCM CD163 & $4.22 \pm 1.30$ & $5.25 \pm 1.28$ & -1.637 & 0.123 \\
\hline PCM CD57 & $29.96 \pm 14.61$ & $53.33 \pm 19.88$ & -2.784 & 0.014 \\
\hline
\end{tabular}

Statistically significant difference $(\mathrm{P}<0.05)$. GLM, granulomatous lobular mastitis; PCM, plasma cell mastitis. 
Table 9 Comparison between the expressions of CD68 and CD163 (GLM group only) and IgG4 (GLM and PCM groups) in patients with and without nipple retraction

\begin{tabular}{|c|c|c|c|c|c|c|}
\hline Items & \multicolumn{2}{|c|}{ No nipple retraction } & \multicolumn{2}{|c|}{ Nipple retraction } & Z & $\mathrm{P}$ \\
\hline GLM CD68 & $4.03 \pm 1.78$ & 4.00 & $5.43 \pm 1.62$ & 6.0 & -1.757 & 0.079 \\
\hline GLM CD163 & $4.14 \pm 1.27$ & 4.0 & $4.71 \pm 1.38$ & 4.0 & -0.668 & 0.504 \\
\hline GLM IgG4 & $5.52 \pm 5.70$ & 5.00 & $12.09 \pm 7.41$ & 12.8 & -2.179 & 0.029 \\
\hline
\end{tabular}

Statistically significant difference $(\mathrm{P}<0.05)$. GLM, granulomatous lobular mastitis; PCM, plasma cell mastitis.

Table 10 Comparison between the expressions of CD68 and CD163 (PCM group only) and CD57 (GLM and PCM groups) in patients with and without nipple retraction

\begin{tabular}{|c|c|c|c|c|}
\hline Items & No nipple retraction & Nipple retraction & $\mathrm{F}$ & $P$ \\
\hline PCM CD68 & $4.00 \pm 2.00$ & $5.00 \pm 1.36$ & -1.076 & 0.299 \\
\hline PCM CD163 & $5.00 \pm 1.00$ & $4.64 \pm 1.45$ & 0.402 & 0.693 \\
\hline PCM CD57 & $41.13 \pm 24.88$ & $40.91 \pm 20.66$ & 0.016 & 0.987 \\
\hline
\end{tabular}

Statistically significant difference $(\mathrm{P}<0.05)$. PCM, plasma cell mastitis; GLM, granulomatous lobular mastitis.

Table 11 Comparison between the expressions of CD163 and IgG4 in GLM patients with different lesion ranges

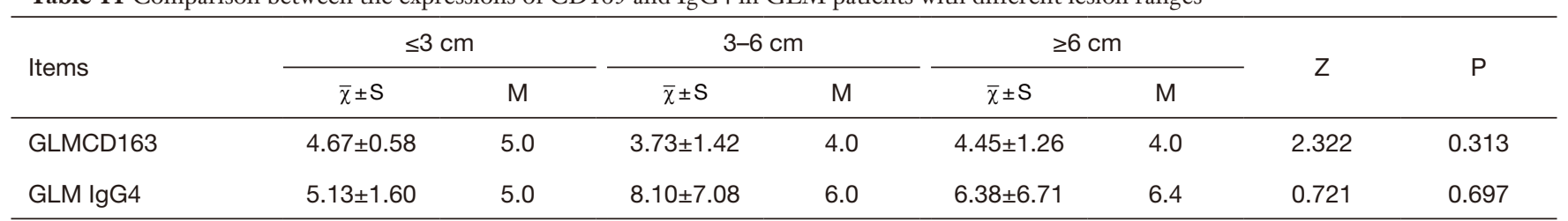

Statistically significant difference $(\mathrm{P}<0.05)$. GLM, granulomatous lobular mastitis.

Table 12 Comparison between the expressions of CD163 and IgG4 (PCM group only) and CD68 and CD57 (GLM and PCM groups) in patients with different lesion ranges

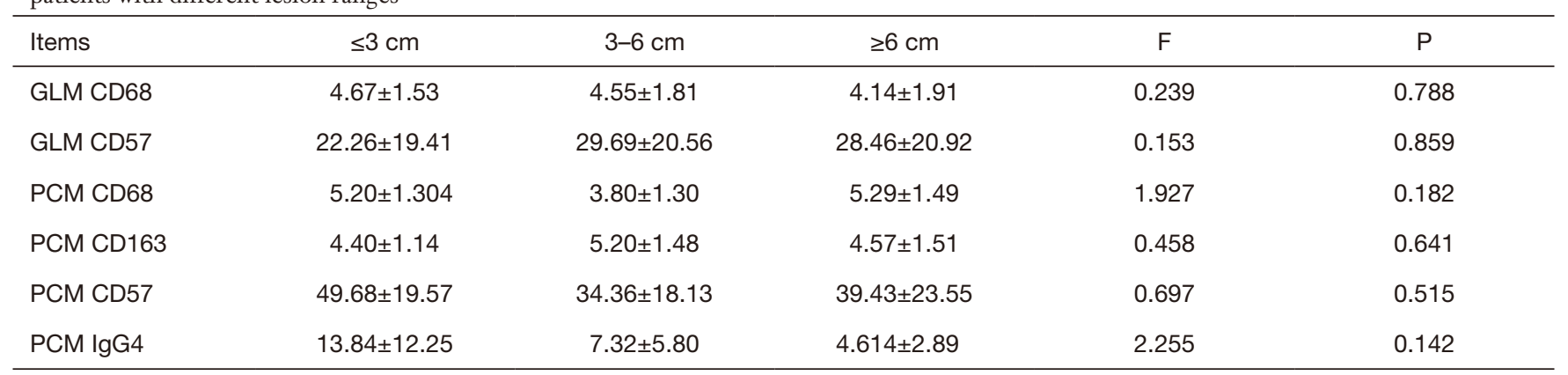

Statistically significant difference $(\mathrm{P}<0.05)$. PCM, plasma cell mastitis; GLM, granulomatous lobular mastitis. 
Cohen (19) proposed that the disease might be a focal lesion of autoimmune disease. A number of domestic studies have confirmed that non-lactational mastitis patients exhibit different degrees of immune dysfunction. Chen et al. reported that the percentage of CD3 and CD4/ $\mathrm{CD} 8$ cells in each subgroup of $\mathrm{T}$ cells were increased, as were the levels of IgG, IGLM, and IgA, and C4, while complement C3 was decreased (20). Xia et al. believed that about $50 \%$ of the patients showed abnormalities in various subsets of T lymphocytes, which were mainly decreased. Immunoglobulin was increased to varying degrees, and complement disorder was more prominent in the decrease of C4 level (9). The two runs in opposite directions, which may be caused by inconsistency of specimens, inconsistency of composition ratio between GLM, PCM, and Zuska disease, or inconsistency of disease course. However, both cellular immunity and humoral immunity are acknowledged to be involved in the disease process. Some studies believe that early application of immunosuppressive agents, methotrexate, and other treatments is effective (21-23). It has been suggested that the disease may be related to autoimmunity. Pathological examination is the gold standard for diagnosis. Currently, there is no unified standard for treatment, but most scholars support a comprehensive surgery-based treatment strategy (24). Further comprehensive treatment options include traditional Chinese medicine therapy, glucocorticoid therapy, immunosuppressive therapy, endocrine therapy, and anti-tuberculosis therapy.

\section{Macrophages}

There are two types of macrophages (25). Classically activated macrophages, also called M1 macrophages, activate immune cells to kill microbes and tumor cells, and a large number of toxic intermediates, presenting antigen to T cells. M1 macrophages protect the body from infection and have a tumor-killing effect. The second type, activated macrophages, also known as M2-type macrophages, produce a large number of cytokines with an antiinflammatory response, mainly to reduce inflammation and promote tissue repair function (26). Maintaining a balance between pro-inflammatory response and anti-inflammatory response can maintain the homeostasis within the internal environment of the body.

CD68 is a macrophage surface marker molecule, and CD163 is a M2-specific macrophage surface marker molecule. One of the important features of chronic inflammatory response is mononuclear/macrophage activation, and a large amount of macrophage infiltration can be seen in the pathological samples from GLM patients. In the early stage, GLM can be treated with cortisol, and so some patients manage to avoid surgical treatment. However, its immune function is suppressed during chronic stress (27). The results of this experiment showed M2-type macrophage infiltration in both granulomatous mastitis and plasmacyte mastitis, suggesting that GLM and PCM may have the same immune activation pathway, and share the same tumorigenesis mechanism. The expression level of macrophages in GLM chronic stress was not significantly different compared with that in acute stress, which suggests that low-dose glucocorticoid therapy in the GLM acute phase may prove effective. In the chronic stage, blocking the M1-type macrophage transformation pathway to M2type macrophages or inhibiting the function of M2-type macrophages may inhibit their tumorigenesis activity, directly affecting the scope of surgery and decreasing the post-GLM damage rate. In clinical application, some GLM patients receive three anti-TB drug treatments in patients with effective, some patients early glucocorticoid treatment effective, but this does not apply to all patients, the long-term curative effect is uncertain, and there is no medical evidence to support this treatment. We speculate that its effect may be related to the enhanced ability of macrophages clear, phagocytosis, and secrete.

\section{NK cells}

NK cells are important members of the body's first line of "ubiquitin-specific" defense against high-risk pathogenic factors (28). They participate in anti-tumor and anti-viral activity, as well as immune regulation, but an imbalance in their activity (hyper or hypoactive) plays a part in diseases such as hypersensitivity and autoimmune diseases. Studies have shown that mature CD57 + NK cells may lead to innovative strategies to prevent human immunosenescence and/or various chronic diseases (29). NK cells secrete stimulant cytokines and inhibitory cytokines to participate in the regulation of early natural immune response and acquired immune response, which is the bridge between natural immunity and acquired immunity $(30,31)$. Liang et al. (32) proposed that different expression patterns of tumor-associated antigens (TAAS) were associated with positive tumor differentiation, small tumor size, and a higher rate of CD57-positive NK cell infiltration. As GLM is a tumorigenic disease, by evaluating the expression 
disorder level of some of the above inflammatory factors, we speculated that the expression level of CD57-positive NK cells in GLM patients might be related to the disease process. The results of this experiment showed that the expression level of CD57 in the GLM group increased as the disease progressed, reaching a peak between 2 weeks and 3 months, while no such change was observed in the PCM group, suggesting that CD57-positive NK cells may be important immune molecules in distinguishing GLM from GLM. In addition, the NK cell threshold may be an important clinical cutoff point for GLM. Regulating the function of NK cells or stimulating cytokines and inhibiting cytokines secreted by NK cells may be essential for the clinical remission or cure of the disease. The expression level of NK cells increased in the group with onset $>2$ years after delivery, suggesting that the occurrence of PCM may be correlated with pregnancy or lactation. Postpartum maternal immune rebound (the dominant Th2 cellmediated immune type in pregnancy is transformed to Th1 cell-mediated immune dominance after childbirth) is considered to be responsible for the aggravation of many autoimmune diseases (33). The incidence of non-lactational mastitis may be related to autoimmunity, so it is necessary to continue to pay attention to the changes of immune indexes in non-lactating mastitis patients 2 years after delivery.

\section{IgG4}

IgG4 is a Th2 cell-dependent globulin, the Fc segment of which can bind to mast cells and participate in the body's allergic reaction process (34). Various inflammatory diseases, such as autoimmune pancreatitis, retroperitoneal fibrosis, sclerosing cholangitis, and a variety of inflammatory pseudotumors, are believed to be caused by IgG4-associated autoimmune disorders (35-38). There have been reports of IgG4-related inflammatory pseudotumors in the breast (39), in which numerous plasma cells showed positive IgG4 in immunohistochemical staining, and patients' serum IgG4 concentration increased. IgG4 may be a useful indicator of inflammatory pseudotumors of the mammary gland, which may benefit from hormone therapy. GLM and the clinicopathological features of breast inflammatory pseudotumors are similar, which may be associated with IgG4. Ogura, Cheuk et al. $(13,40)$ think granulomatous mastitis can be divided into IgG4-related and non-IgG4related granulomatous lobular mastitis: the former is associated with pregnancy, while the latter is associated with autoimmune diseases, for which steroid hormone treatment can be effective. This helps some patients to avoid postoperative mammary gland damage, which is beneficial to their psychological recovery.

IgG4-related sclerosing mastitis (IgG4-SM) is a special type of mammary gland inflammation. Some scholars have raised the question whether some cases of granulomatous lobular mastitis can be classified as IgG4-SM. According to one study (40), 9 cases of lymphocytic mastitis had no IgG4-positive plasma cell infiltration and 6 of 7 cases granulomatous lobular mastitis had no IgG4-positive plasma cell infiltration. However, the relatively small number of cases in the study makes it difficult to conclude whether granulomatous mastitis bears a correlation with IgG4related sclerosing mastitis. The results of this experiment showed that generally there was a low expression of IgG4 in GLM and PCM, and its expression level was consistent. However, the expression of IgG4 differs depending on whether GLM is combined with nipple depression. The expression of IgG4 in GLM with nipple depression is significantly increased, which suggests that the two groups may in fact be different clinical subtypes of GLM, and have a different pathogenesis. The expression level in patients with inverted nipples was significantly higher than in those with non-inverted nipples, which indicates that the increase of IgG4 may be related to the blockage of the milk duct, damage to the skin cells in the milk duct, and the immune response induced by the exposure of autoantigens. To some extent, this is consistent with the idea that GLM can be divided into IgG4-correlated and non-IgG4-correlated. However, there was no significant correlation between the expression of $\operatorname{IgG} 4$ in patients and fertility status and time since the end of the last lactation period. Based on the above, GLM can be separated into IgG4-correlated and non-IgG4-correlated. The former is related to nipple depression, while the latter is not. In addition, the low expression level of IgG4 in GLM or PCM did not meet the diagnostic criteria for IgG4-related diseases and were not part of the categorization of this disease. Therefore, the expression level of IgG4 did not provide theoretical support for the use of immunosuppressive therapy.

\section{Conclusions}

In conclusion, both GLM and PCM are immune-related diseases, and various immune cells and cell components are involved in their occurrence and development. However, there are differences between the two diseases. M2-type macrophages play a major role in the course of GLM 
and PCM, and are closely related to tumor formation. CD57-positive NK cells may offer an important basis for differentiation between GLM and PCM, and may also serve as an important dividing point for the progression of GLM, which is of great significance for guiding appropriate treatment. Inverted nipples are an essential basis for differentiating between IgG4-related and nonIgG4-related GLM; and the mechanism in the pathogenesis and immune mechanism of these two disease may differ, and the treatment methods required may also differ. At present, GLM and PCM are mainly treated through a comprehensive surgical approach. The results of this study can prove that a small number of clinical GLM patients can benefit from immunosuppressive therapy and avoid surgery. The pathogenesis of non-lactational mastitis, such as GLM, needs to be confirmed based on further basic and clinical application research.

Of course, this experiment still had many limitations. First, during the study period (one year), the number of PCM outpatients and inpatients was low, and none of the patients with cystic breast cystic hyperplasia required biopsy or surgery, which resulted in difficulty in obtaining sampling specimens. The sample sizes of the two control groups were relatively small, which may have created bias in the results. Future studies will include larger sample sizes. Second, no further experiments were conducted to detect the expression of these indicators (CD68, CD163, CD57, and IgG4) in human serum, and its etiology and pathogenesis are still not clear. Third, it is better to identify NK cells or macrophages with immunofluorescence staining than immunohistochemistry. In this study, no immunofluorescence staining was performed on the samples, which may have led to biased results. Other more sensitive methods will be used to detect macrophages and NK cells in future studies. Fourth, no animal experiments were conducted, and no feasible suggestions for its treatment were proposed. Last, because mammary gland tuberculosis case is rare, the control group offered no specific mammary inflammatory disease comparison. The control group would have better served its purpose made up of patients with a specific inflammatory disease of the breast (tuberculosis of the breast).

\section{Acknowledgments}

We would like to thank the patients that participated in this study.

Funding: This study was supported by Key Projects of China
Hunan Provincial Science \& Technology Department (No.2017SK2142).

\section{Footnote}

Data Sharing Statement: Available at http://dx.doi. org/10.21037/gs-20-419

Conflicts of Interest: All authors have completed the ICMJE uniform disclosure form (available at http://dx.doi. org/10.21037/gs-20-419). All authors report grants from Key Projects of China Hunan Provincial Science \& Technology Department, outside the submitted work.

Ethical Statement: The authors are accountable for all aspects of the work in ensuring that questions related to the accuracy or integrity of any part of the work are appropriately investigated and resolved. The trial was conducted in accordance with the Declaration of Helsinki (as revised in 2013) and the Harmonized Tripartite Guideline for Good Clinical Practice from the International Conference on Harmonization. This study was reviewed and approved by the Hamilton Integrated Research Ethics Board (approval \#2017SK2142) and the Hoffmann-La Roche global review committee. All patients enrolled completed the informed consent form.

Open Access Statement: This is an Open Access article distributed in accordance with the Creative Commons Attribution-NonCommercial-NoDerivs 4.0 International License (CC BY-NC-ND 4.0), which permits the noncommercial replication and distribution of the article with the strict proviso that no changes or edits are made and the original work is properly cited (including links to both the formal publication through the relevant DOI and the license). See: https://creativecommons.org/licenses/by-nc-nd/4.0/.

\section{References}

1. Renshaw AA, Derhagopian RP, Gould EW. Cystic neutrophilic granulomatous mastitis: an underappreciated pattern strongly associated with gram-positive bacilli. Am J Clin Pathol 2011;136:424-7.

2. Kieffer P, Dukic R, Hueber M, et al. A young woman with granulomatous mastitis: a corynebacteria may be involved in the pathogenesis of these disease. Rev Med Interne 2006;27:550-4.

3. Bogomolov DV, Dolzhanskii OV. Chronic granulomatous 
mastitis in the practice of pathologists. Arkh Patol 2005;67:25-9.

4. Tedeschi LG, Ouzounian G, Byrne JJ. The role of ductal obstruction and hormonal stimulation in mammary duct ectasia. Surg Gynecol Obstet 1962;114:741-4.

5. Going JJ, Anderson TJ, Wilkinson S, et al. Granulomatous lobular mastitis. J Clin Pathol 1987;40:535-40.

6. Fletcher A, Magrath I, Riddell R, et al. Granulomatous mastitis: a report of seven cases. J Clin Pathol 1982;35:941-5.

7. Song A. Granulomatous mastitis in 52 cases of clinical retrospective analysis: Keep pace with The Times of breast epidemiology. The 12th national Chinese medicine, traditional Chinese and western medicine of traditional Chinese medicine combined with breast disease academic conference, Beijing, China, 2011;234-1.

8. Zhang C, Lei S, Kong C, et al. Clinical study on surgical treatment of granulomatous lobular mastitis. Gland Surg 2019;8:712-22.

9. Zhou F, Yu L, Ma Z, et al. Granulomatous lobular mastitis. Chronic Dis Transl Med 2016;2:17-21.

10. Xia Y, Chen H, Ye M, et al. Variations of T lymphocytes, immunoglobulin, and complements levels in peripheral blood of non-lacta. Chin J Breast Dis (Electronic Edition) 2012;5:504-14.

11. Shao $\mathrm{X}, \mathrm{Wu} \mathrm{B}, \mathrm{Cheng} \mathrm{L}$, et al. Distinct alterations of CD68+CD163+ M2-like macrophages and myeloidderived suppressor cells in newly diagnosed primary immune thrombocytopenia with or without CR after highdose dexamethasone treatment. J Transl Med 2018;16:48.

12. Ogura K, Matsumoto T, Aoki Y, et al. IgG4-related tumour-forming mastitis with histological appearances of granulomatous lobular mastitis: comparison with other types of tumour-forming mastitis. Histopathology 2010;57:39-45.

13. Zhang Y, Yen W, Li Q, et al. Stents method tit diorthosis postoperative nursing function analysis. Chongqing Medical 2016;45:95-6.

14. Su L. Expression of CD3, CD20, CD68 and immune mechanism in plasma cell mastitis. Ningxia medical university, 2009.

15. Bässler R. Mastitis. Classification, histopathology and clinical aspects. Pathologe 1997;18:27-36.

16. Zhang C. Introduction: the progress in the diagnosis and treatment of lactation mastitis: controversy and consensus. Medicine and Philosophy 2013;34:7.

17. Baslaim MM, Khayat HA, Al-Amoudi SA. Idiopathic granulomatous mastitis: a heterogeneous disease with variable clinical presentation. World J Surg
2007;31:1677-81.

18. Yang X, Zhang Y, Wang Y, et al.Granulomatous mastitis pathogenesis to discuss:11 th national Chinese medicine and traditional Chinese and western medicine combined with mammary gland disease academic conference, China's Guangxi Guilin 2009;379-81.

19. Cohen C. Granulomatous mastitis. A review of 5 cases. S Afr Med J 1977;52:14-6.

20. Chen F, Feng J, Gao Q, et al. Non breast-feeding mastitis patient peripheral immune function test and its clinical significance. Theory and Practice of Surgery 2015;20:252-4.

21. DeHertogh DA, Rossof AH, Harris AA, et al. Prednisone management of granulomatous mastitis. N Engl J Med 1980;303:799-800.

22. Raj N, Macmillan R, Ellis I, et al. Rheumatologists and breasts: immuno suppressive therapy for granulomatous mastitis. Rheumatology 2004;43:1055-6.

23. Kim J, Tymms KE, Buckingham JM. Methotrexate in the management of granulomatous mastitis. ANZ J Surg 2003;73:247-9.

24. Xiao Q, Zhang C. Modern research progress of non breast-feeding mastitis. Medicine and Philosophy 2013;34:8-13.

25. Gordon S. Macrophage heterogeneity and tissue lipids. J Clin Invest 2007;117:89-93.

26. Wynn TA, Barron L, Thompson RW, et al. Quantitative assessment of macrophage functions in repair and fibrosis. Curr Protoc Immunol 2011; Chapter 14:Unit14.22.

27. Dhabhar FS. Acute stress enhances while chronic stress suppresses skin immunity. The role of stress hormones and leukocyte trafficking. Ann N Y Acad Sci 2000;917:876-93.

28. Deng Y, Kerdiles Y, Chu J, et al. Transcription factor Foxo1 is a negative regulator of natural killer cell maturation and function. Immunity 2015;42:457-70.

29. Kared H, Martelli S, Ng TP, Pender SL, Larbi A. CD57 in human natural killer cells and T-lymphocytes. Cancer Immunol Immunother 2016;65:441-52.

30. Sawaki J, Tsutsui H, Hayashi N, et al. Type 1 cytokine/ chemokine production by mouse NK cells following activation of their TLR/MyD88-mediated pathways. Int Immunol 2007;19:311-20.

31. Newman KC, Riley EM. Whatever turns you on: accessory-cell-dependent activation of NK cells by pathogens. Nat Rev Immunol 2007;7:279-91.

32. Liang J, Ding T, Guo Z, et al. Expression pattern of tumour-associated antigens in hepatocellular carcinoma: association with immune infiltration and disease 
progression. Br J Cancer 2013;109:1031-9.

33. Piccinni MP, Scaletti C, Maggi E, Romagnani S. Role of hormone-controlled Th1- and Th2-type cytokines in successful pregnancy. J Neuroimmunol 2000;109:30-3.

34. He Z, Tang G, Lu Q, et al. IgG4 serum levels in the diagnosis of IgG4 related diseases and rheumatic autoimmune disease application value in the differential diagnosis. The Chinese medical association seventh national inspection of middle-aged and young medical academic conference, nanjing, jiangsu province, China 2012;153-4.

35. Kamisawa T, Funata N, Hayashi Y, et al. A new clinicopathological entity of IgG4-related autoimmune disease. J Gastroenterol 2003;38:982-4.

Cite this article as: Kong C, Zhang C, Wu Y, Zeng Z, Yu H, Zeng J, Lei S, He J, Fan P. The expression and meaning of CD68, CD163, CD57, and IgG4 in granulomatous lobular mastitis. Gland Surg 2020;9(4):936-949. doi: 10.21037/gs-20-419
36. Sima P, Vannucci L, Vetvicka V. Atherosclerosis as autoimmune disease. Ann Transl Med 2018;6:116.

37. Okazaki K, Chiba T. Autoimmune related pancreatitis. Gut 2002;51:1-4.

38. Kamisawa T, Funata N, Hayashi Y, et al. Close relationship between autoimmune pancreatitis and multifocal fibrosclerosis. Gut 2003;52:683-7.

39. Zen Y, Kasahara Y, Horita K, et al. Inflammatory pseudotumor of the breast in a patient with a high serum IgG4 level: histologic similarity to sclerosing pancreatitis. Am J Surg Pathol 2005;29:275-8.

40. Cheuk W, Lee K C, Chong L, et al. IgG4-related Sclerosing disease: a potential new etiology of cutaneous pseudolymphoma. Am J Surg Pathol 2009;33:1713-9. 